\title{
Based on Wide Area Environment Abnormal Behavior Analysis and Anomaly Detection Research
}

\author{
Lin Zhang ${ }^{1}$ Xiaoping $\mathrm{Li}^{2}$, \\ ${ }^{1}$ Beijing University of Civil Engineering and Architecture, Beijing, China \\ ${ }^{2}$ Beijing Institute of Technology, Beijing, China
}

\begin{abstract}
Group anomaly identification and location is an important issue in the field of artificial intelligence. Capture of the accident source and rapid prediction of mass incidents in public places are difficult problems in intelligent video identification and processing, but the traditional group anomaly detection research has many limitations when it comes to accident source detection and intelligent recognition. We are to research on the algorithms of accident source location and abnormal group identification based on behavior analysis in the condition of dramatically changing group geometry appearance, including: 1) to propose a logic model of image density based on the social force model, and to build the crowd density trend prediction model integrating "fast and fuzzy matching at front-end" and "accurate and classified training at back-end"; 2) to design a fast abnormal source flagging algorithm based on support vector machine, and to realize intelligent and automatic marking of abnormal source point; 3 ) to construct a multi-view human body skeleton invariant moment model and a motion trajectory model based on linear parametric equations. The expected results of the research will help prevent abnormal events effectively, capture the first scene of incidents and the abnormal source point quickly, and play a decision support role in the proactive national security strategy.
\end{abstract}

\section{Introduction}

Some large-scale unexpected mass incidents occur more frequently in public area with the development of society and economy and growth of population density. These things will lead to serious consequences, if we can't capture the first scene and the source point quickly and do the rapid early warning.

The existing video surveillance system is lack of intelligence. Its real time performance of intelligent decision for group abnormal events is poor. It is always limited to fixed single camera which can only distinguish and determine some simple abnormal behavior and it is lack of recognition of the high level complex behavior of the human body. People need to find out the abnormal source from the massive video after the abnormal event occurs and this can't meet the real-time requirements of emergency management of major events. The existing monitoring system must be transformed from close to the Internet to improve the collaborative analysis of video surveillance system and the ability of group anomaly detection and localization and the working efficiency of the camera network. This paper is intended to study the detection of abnormal source points of the wide area group and the determination of the nature of the source points based on the understanding and analysis of the intelligent video, try to make breakthroughs on abnormal population model, abnormal source detection and location, the source of abnormal attribute analysis, model training and matching, camera collaborative scheduling key technology. In order to achieve population density trends prediction of according to the "fast fuzzy matching" and "accurate classification of training", group trend prediction should be combined with social force model and population density trend model should be built, anomaly source should be focused by multi stage split control strategy to capture the first scene of the abnormal events of the source point timely and predict the trend of group events rapidly. Rapid propagation of new abnormal group model, multi region and multi scene linkage realtime warning should be achieved by continuous training "group trend - the source of the object behavior attribute" relationship, determining attribute of abnormal source and pushing analysis on the attribute of the anomaly source. This will make a positive contribution to the early warning of public places.

\section{Theoretical basis research}

In view of the above questions, we analyzed the population density estimation research, intelligent video surveillance, moving object behavior analysis, and machine learning based on support vector machine and pattern classification methods. 


\subsection{Analysis of group event types}

Intelligent video surveillance [1], become one of the hot research fields of artificial intelligence, has been transformed from the "passive monitoring" to "active control". Group of abnormal behavior recognition technology driven by requirements has attracted more and more attention of researchers. The U.S. Defense Advanced Research Projects Agency has set up a major project of visual surveillance VSAM which is mainly used for the analysis of crowd behavior in battlefield environment and civil video monitoring scene. Davies is the first to apply the crowd density monitoring system to the subway station in London using background subtraction and edge detection method to calculate the number of pixels. Ramin Mehran is the first to propose the method using the social force model to detect anomaly, use the optical flow method to extract the dynamic information of the image in the crowd and use Newtonian mechanics to characterize the individual and mutual movement. Saira Saleem Pathan is the first to use social entropy to quantify the uncertainty of potential field and use SVM to detect the crowd abnormal. However, the real-time detection of abnormal group still faces a series of technical problems, for example, the traditional video monitoring system still can't confirm the category of abnormal alarm event and information of abnormal source. The proper descriptive Characteristics of moving crowd must be found, a reliable motion model should be established, analysis and position of abnormal crowd should be realized in order to reduce the space dimension and improve processing speed.

\subsection{Analysis of anomaly source location}

At present, there isn't any research that studies the location of abnormal source and judgment of anomaly types, causes and attribution under the group abnormal environment. The logical relation between the abnormal area and the abnormal source should be established according to different group movement models because of complex variability of population movement, the motion model should be classified by mass samples of moving groups that are collected by the front end and a special group abnormal behavior model should be trained using SVM. The biggest advantage of SVM is that it does not need to reduce the dimension of data when it is classified and it has good performance in terms of convergence, training speed, generalization, and classification accuracy Rabaud and Belongie [2] proposed Kanade-Lucas-Tomasi tracking algorithm tracking the target by clustering similar features on the track. Chow estimated the population density based on global hybrid neural network learning algorithm. He got pixel statistics in image sequences, then estimated population density using neural network modeling after the study. Donatell found a method which could use SURF algorithm to extract the image feature points and use the support vector machine to train some selected samples, then count population density of scene through support vector machine. This paper proposes a feature representation based on the video image partition region density calibration and scalar, identifies the abnormal source in the abnormal group by semiautomatic and screens a more effective training set through continuous training and learning, then finds out the relationship between the population trend characteristics and the distribution of abnormal source points. At the same time it also realizes the all-time intelligent identification of abnormal source points using appropriate training machine kernel function and adopting moment invariant adaptive strategy considering the multi angle and multi modal changes in video. image.

\subsection{Analysis of the behavior of abnormal objects}

Behavioral attribute analysis has a wide range of applications, such as intelligent monitoring, humancomputer interaction, training of athletes, video coding, etc. Many scholars have put forward a lot of behavior analysis algorithms to identify the complex event and individual behavior. Efros et al. poposed the characteristics of the optical flow of the low precision motion image using the fuzzy optical flow feature. Dalal et al. ${ }^{[3]}$ analyze human using the differential optical flow histogram and gradient direction histogram as input features. Alberto et al. tried to recognize the human body posture using the fuzzy finite state machine. Vajda et al. used dynamic time warping method in motion matching and this method has the characteristics of time sequence, robustness and good performance. ZhiZhongused L-K algorithm to capture the optical flow information proposed by BruceD [4]. Lucas to get the image of the energy flow value and got an intuitive reflection of the dynamic changes of the target [5]. Billing et al. proposed two algorithms based on PSL (Predictive Sequence Learning) [6]. Lin et al. analyzed the principal component of video frames and then classified and identified the sequence of events, but the large amount of data of the image led to a very large amount of computation. Jacob combined the assumption of projection, context dependent reasoning, and structured HMM to identify the expected behavior in the GMT (Ground Motion Target Indicator) data. Crowd control system should be able to provide early warning of the occurrence of abnormal behavior in the video environment. Motion trajectory could be obtained by using the moving human tracking algorithm and the abnormal behavior could be estimated by extracting the length, pixel, covariance, motion degree and other information from the trajectory [7].The study of behavioral analysis mainly focuses on the behavior identification of a limited class of simple rules or abnormal behavior detection of single target in a particular scene [8], complex and large amount of calculation of the image processing should be carried out before behavioral analysis, this reduces the efficiency of the algorithm and it's difficult to achieve intelligent frontend equipment. Most of the algorithms can only achieve the static matching of single target behavior in the process of analysis and the capture efficiency is low.

\section{Program architecture design}


In order to analyze the group abnormal behavior and determine the abnormal source object attribute, we combine the social force model, the trend of group movement and density information, divide the Video frames into different region through the grid area scan, predict the speed and direction of population movement based on crowd time domain trajectory logic model and carry on the fuzzy matching of anomaly groups based on the crowd spatial domain density logic model, then give the predictive result by focusing on the source points and analyzing the source object attribute. The flowchart as shown in Fig. 3.1.

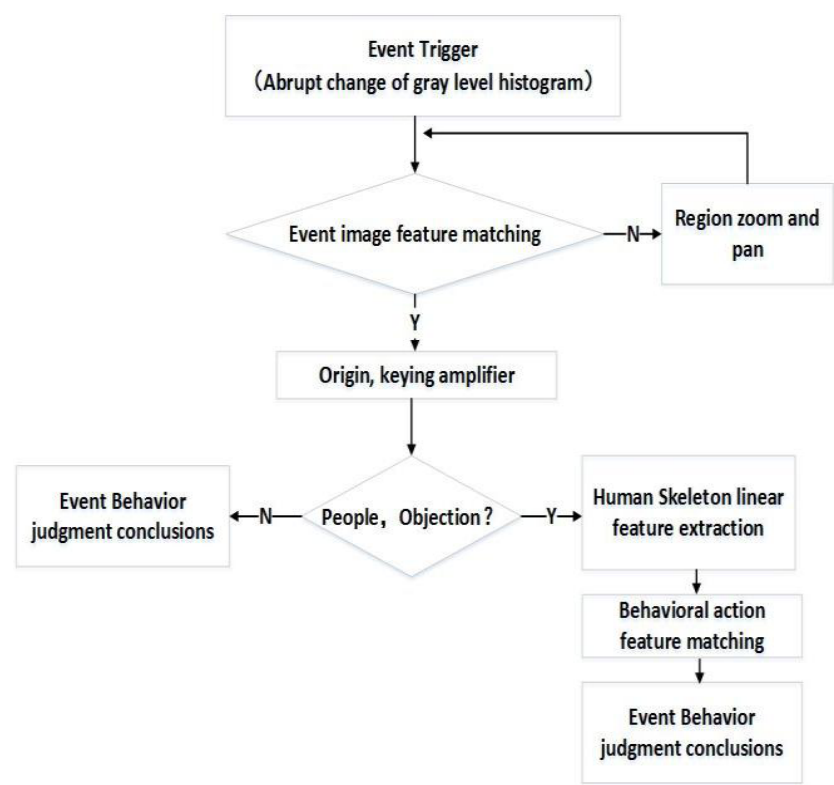

Figure 1. The process of the group abnormal behavior prediction and the source point attribute analysis.

\section{Conclusions}

Aiming at the problems of marking groups abnormal source and analyzing source behavior under intelligent video surveillance, the fast feature matching of the sudden mass events in public places and the first time to capture the abnormal object are realized by establishing of the model which is related to the change tendency of the group characteristic and the abnormal character of the source point; the fast algorithm designed should be focused on rapid detection of abnormal population in complex background, abnormal target location, Characteristic model of population movement tendency in the series of basic problems of abnormal behavior analysis, adaptive SVM training, analysis of abnormal source and image transmission coding protocol in wide area environment.

The suspected abnormal points is located through the early abnormal group area detection and locked combining with the lens multi-level collaborative scheduling algorithm, the model of "group individual" feature is established according to the change characteristic of abnormal group and the position information of abnormal point.

\section{References}

1. Zhang $\mathrm{P}$, Zhang $\mathrm{X}$, Li J, et al. The effects of temperature and salinity on the swimming ability of whiteleg shrimp, Litopenaeus vannamei[J]. Comparative Biochemistry and Physiology, Part A: Molecular \& Integrative Physiology, 2007, 147(1): 64-69.

2. Vapnik V N.The Nature of Statistical Learning Theory[M].Springer, 1995.

3. Dalal N, Triggs B. Histograms of oriented gradients for human detection[C]/Proceedings of the Conference on Computer Vision and Pattern Recognition(CVPR'05). 2005,1:886-893.

4. Bruce D, Lucas, Takeo Kanade. An Iterative Image Registration Technique with an Application to Stereo Vision[C]. Proceedings of the 7th International Joint Conference on Artificial Intelligence. 2006(11): 674679.

5. Billing, E.A, Hellstro, Janlert, L.-E. Behavior recognition for Learning from Demonstration[C]. 2010 IEEE International Conference on Robotics and Automation (ICRA), 2010(5): 866-872.

6. Lin Chunli, Wang Kejun. A behavior classification based on Enhanced Gait Energy Image[C]. 2010 2nd International Conference on Networking and Digital Society (ICNDS). 2010(5): 589- 592.

7. Lee J J, Kim G J, Kim M H. Trajectory extraction for abnormal behavior detection in public area[C]//Emerging Technologies for a Smarter World (CEWIT), 2012 9th International Conference \& Expo on. IEEE, 2012: 1-5.

8. Zhong Hua, Shi Jian-bo, Visontai M. Detecting unusual activity in video[A]. In: Proceedings of IEEE Conference on Computer Vision and Pattern Recognition[C], Washington DC, USA, 2004:819826. 